\title{
Effects of whole body vibration training and mental training on mobility, neuromuscular performance, and muscle strength in older men
}

\author{
Maryam Goudarzian', Samira Ghavi ${ }^{2}$, Ardalan Shariat', Hossein Shirvani', Mostafa Rahimi ${ }^{5, *}$ \\ IIranian Research Center on Healthy Aging, Sabzevar University of Medical Sciences, Sabzevar, Iran \\ 2Department of Physical Education and Sport Sciences, University of Semnan, Semnan, Iran \\ ${ }^{3}$ Sports Medicine Research Center, Neuroscience Institute, Tehran University of Medical Sciences, Tehran, Iran \\ ${ }^{4}$ Exercise Physiology Research Center, Life Style Institute, Baqiyatallah University of Medical Sciences, Tehran, Iran \\ ${ }^{5}$ Department of Physical Education and Sport Sciences, Faculty of Humanities, Shahrekord University, Shahrekord, Iran
}

This study was designed to evaluate the effects of whole body vibration (WBV) exercise, mental training (MT), and the concurrent effect of WBV and MT on lower body balance, neuromuscular performance, and leg muscle strength in elderly men. In a randomized control trial study with parallel group design, 42 older men (mean \pm standard deviation age, $68 \pm 5.78$ years) were randomly divided into four groups: WBV $(n=11), M T(n=12), W B V+M T(n=10)$, and control $(n=9)$ groups. The protocol of training consisted of three sessions per week for 8 weeks and about $30 \mathrm{~min}$ for each session. The WBV exercise was performed on a vibration machine. In MT group, participants were asked to mentally visualize to do the Timed Up and Go and relaxation technics. postural stability, the Timed Up and Go test, 5-repetition chair-rising test, 6-m tandem gait test, 10-m walking, and leg isometric strength were measured in baseline and after 8-week intervention. Repeated measures-analysis of variance followed by post hoc was used to analyses the data. The results of this study s howed that there were significant improvements $(P<0.05)$ in postural stability, Timed Up and Go, 5-repetition chair-rising, $6-\mathrm{m}$ tandem gait test, 10-m walking, and leg isometric strength in WBV, MT, and WBV+MT in comparison with baseline and in comparison with control group. It seems that older adults can take benefit from WBV and MT and WBV+MT exercise as a cost-effective and practical way without side effects to improve postural control, mobility, and functional performance.

Keywords: Aging, Mobility, Stability, Vibration, Mental training, Muscle strength

\section{INTRODUCTION}

Age-related decrease in muscle mass and strength leads to declined mobility, independence, augmented fall risk, and lessened quality of life (Bogaerts et al., 2007a). Some of the risk factors, including loss of muscle strength and muscle power in lower extremities, balance and postural control as well as functional mobility have been recognized as responsible for the increase in chances of falling (Osugi et al., 2014). Therefore, finding the practical way to prevent and treat this ailment, specifically concerning improvements in balance and postural control seems necessary.
It is confirmed that a different kind of exercise, such as aerobic and resistance exercises as well as tai-chi have effectively increased physical fitness and decreased the chances of falling among older people (Von Stengel et al., 2012). However, due to other age-related restrictions, such as cardiac limitation, weak balance or absence of enough motivation or willingness, many people are incapable of, or reluctant to do common and traditional exercises. Therefore, researchers have been looking for new approaches that are, first and foremost, suitable for enhancing physical fitness and neuromuscular performance and are consequently feasible and comfortable for older people. Specifically, whole body vibration (WBV)
${ }^{*}$ Corresponding author: Mostafa Rahimi (D) https://orcid.org/0000-0002-1592-2601 Department of Physical Education and Sport Sciences, Faculty of Humanities, Shahrekord University, Rahbar Blvd., Shahrekord 88186/34141, Iran Tel: +98-9132811620, Fax: +98-3155912543, E-mail: Mostafa.rahimi20@gmail.com Received: June 20, 2017 / Accepted: September 29, 2017
This is an Open Access article distributed under the terms of the Creative Commons Attribution Non-Commercial License (http://creativecommons.org/licenses/by-nc/4.0/) which permits unrestricted non-commercial use, distribution, and reproduction in any medium, provided the original work is properly cited. 
and mental training (MT) have been receiving attention in recent years, especially in the area of elderly empowerments (Osugi et al., 2014). In this context, previous studies have examined the benefits of WBV exercise on muscle mass and strength, balance, mobility, and functional performance among both healthy and frail elderly patients (Adsuar et al., 2012; Bogaerts et al., 2011; Verschueren et al., 2004; Zhang et al., 2014). Likewise, meta-analysis papers have reported that WBV exercise can significantly improve leg muscle strength (Lau et al., 2011) as well as balance and functional mobility (Adsuar et al., 2012; Hiroshige et al., 2014; Lam et al., 2012; Rogan et al., 2011) in older adults. Moreover, it has been reported that WBV exercise is safe and tolerable for the elderly, with relatively slight side effects (Kawanabe et al., 2007; Verschueren et al., 2004; Zhang et al., 2014). MT, on other hand, is an exercise approach that the elderly or people with physical disabilities can derive benefits from. MT functions through motor imagery being raised as a reconstructed movement in the mind without making a simultaneous sensory input or obvious and visible output, because the same neural mechanisms that participate in learning with physical exercises are activated in MT (Malouin et al., 2010a). MT has many advantages, in that it is simple, safe and economical, requiring no special equipment and facilities, and reliant on simple education, without causing any subsequent fatigue (Hosseini et al., 2011). Since previous studies have suggested that MT exercise, along with traditional exercise, would be more useful for older adults, these exercises can be supplemented with common physical exercise (Beauchet et al., 2010; Fansler et al., 1985; Hamel and Lajoie, 2005; Malouin and Richards, 2010). It has been reported that exercise imagery in older adults have positive effects on falling and improved execution time of Timed Up and Go (TUG) test (Kim et al., 2012), however, there were not priority than physical exercise (Feltz et al., 1985; Salmanian and Farokhi, 2008) and likewise it has been suggested that physical exercise is the most important part of exercise sessions (Batson et al., 2007; Jackson et al., 2004; Linden et al., 1989).

As such, the main aim of this study was to examine the effects of WBV exercise and MT, as well as the concurrent effects of WBV and MT on lower body balance, neuromuscular performance, and leg muscle strength among elderly men, and it was hypothesized that the effects of WBV on lower body balance, neuromuscular performance, and leg muscle strength would be greater than any singular effect of WBV or MT. As the positive effects of WBV and MT are linked to the physical characteristics of the elderly, the novelty of this research is related to the concurrent effects of WBV and MT on selected variables.

\section{MATERIALS AND METHODS}

\section{Participants}

In this study, a randomized controlled trial (IRCT201405181 $7743 \mathrm{~N} 1$ in Iranian registry of clinical trials) with parallel group design was used among 42 healthy old men (mean \pm standard deviation age, $68 \pm 5.78$ years). Participants were recruited by advertisements in parks and public places. This study was started from August 2016 and finalized by the end of October 2016. The physician screened the participants and checked the inclusion and exclusion criteria (Kargarfard et al., 2016). They were excluded if they, (a) were outside the age range of 60 to 85 years; (b) had implants in the lower extremity or the spine, (c) had a current diseases for which exercise is contraindicated; (d) had participated at least 2-3 times/wk in any exercise program in the past year; and (e) had contraindications for participation in WBV (fractures, neuromuscular and heart diseases, stroke, implant, bypass, stent, rheumatic arthritis and other joint disease, epilepsy). Sample size was calculated using the $G *$ Power 3.1 the current power of 0.80 , $\alpha=0.05$, and an effect size of 0.30 . The subjects were randomly divided into four groups: WBV $(\mathrm{n}=11), \mathrm{MT}(\mathrm{n}=12), \mathrm{WBV}+\mathrm{MT}$ $(\mathrm{n}=10)$, and control ( $\mathrm{n}=9)$ groups presented in CONSORT (consolidated standards for reporting of trials) flowchart (Fig. 1). Participation in this study was voluntary and there was no drop out during 8-week research. This study was approved by participants signed an informed consent form in accordance with the ethical guidelines of the Helsinki Declaration pertaining to the use of human participants in medical research. Ethics approval was obtained from the Ethics Committees of Sabzevar University of Medical Sciences $(122 / 9,462)$ on 23 July 2016.

\section{Intervention protocol}

The WBV exercise was performed 3 times a week for 2 months (8 weeks, to be precise) on a vibration machine (Novotec, Pforzheim, Germany). The participants in the WBV group were asked to stand on the oscillating platform, that is associated with peak-to-peak amplitude of 5-8 $\mathrm{mm}$ of vertical vibration, and frequency was set at 30 to $35 \mathrm{~Hz}$. The work-to-rest ratio was 1:1 (6 set of 45 to $80 \mathrm{sec}$ ). The exercise protocol included six positions: (a) standing straight with knees semi-locked; (b) isometric squat at a knee angle of approximately $120^{\circ}$; (c) kneeling on the floor with arms straight and hands placed on the platform; (d) squatting at a rhythm of 2 sec up and 2 sec down at a knee angle of approximately $120^{\circ}$; (e) lunge position with left leg on platform and right leg on ground; and (f) lunge position with right leg on plat- 


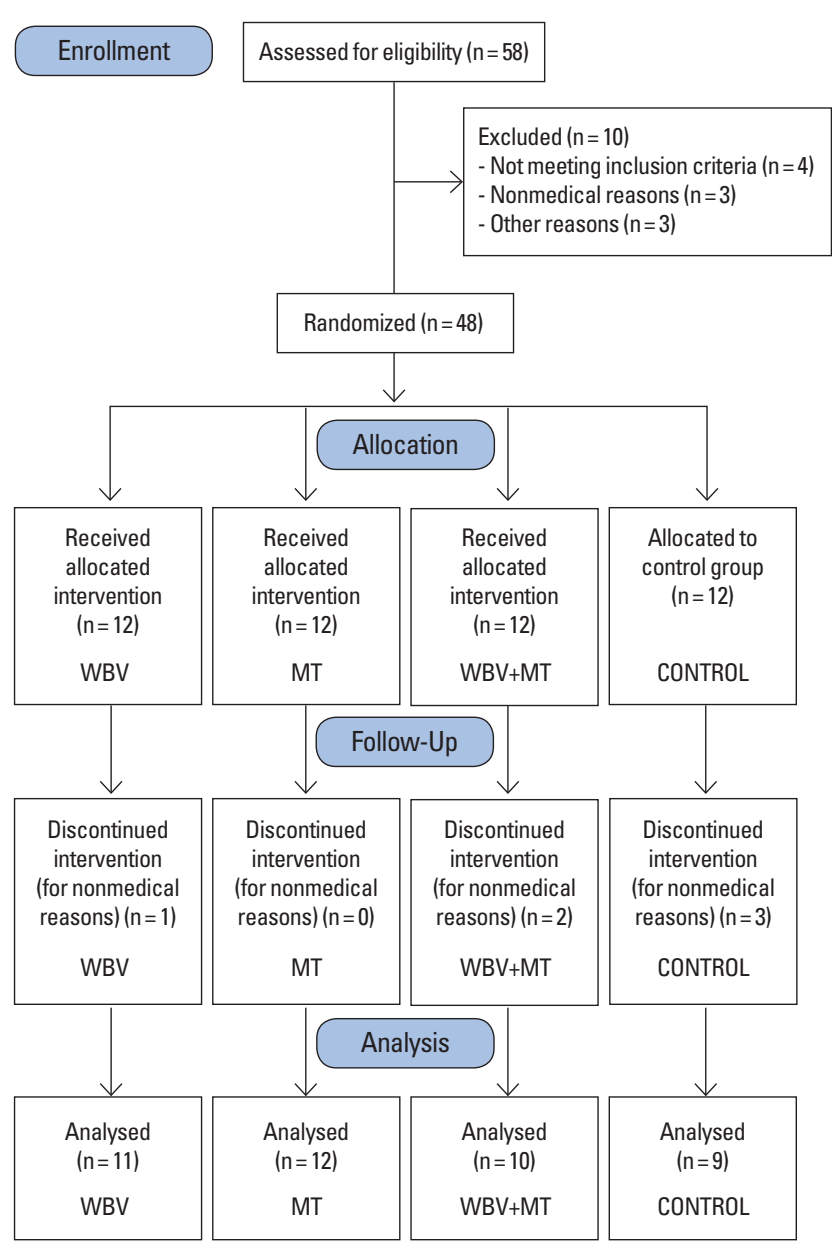

Fig. 1. CONSORT (consolidated standards for reporting of trials) flowchart. WBV, whole body vibration; MT, mental training.

form and left leg on ground (Goudarzian et al., 2017). An experienced personal trainer, certified from American College of Sports Medicine supervised all the exercise sessions.

In the MT group, participants were assembled in an appropriate and quiet room for mental practice from 8 a.m. to 12 noon. The practice time was similar to WBV exercise protocol for each session. In order to increase concentration and readiness, relaxation techniques such as breathing was adjoined for $10 \mathrm{~min}$. Each participant was made to lie down on a flat bed and close his eyes. They were then asked to imagine themselves sitting on an armchair. After that, they were instructed by the trainer to stand up and walk a distance of $3 \mathrm{~m}$ towards a special goal, and then turn back and sit down on the armchair. Next, they were asked to try and mentally visualize the same scenario, this time with more speed and skill in each period (Malouin et al., 2010b).

In the WBV+MT (concurrent) group, the participants were subjected to a combination of vibration and MT that was the halftime of each protocol. In the control group, the participants did not participate in any exercise program and were asked to assume their daily routine throughout the course of the study.

\section{Measurements}

Before commencing on the intervention, the participants' body mass was assessed to the nearest $0.1 \mathrm{~kg}$ and height to the nearest $0.1 \mathrm{~cm}$ (Seca GmbH, Hamburg, Germany), all the while in minimal or lightweight clothing and without any footwear.

Postural Stability was measured by Biodex device in 8-12 levels of difficulty (Balance System SD, Biodex Medical Systems Inc., Shirley, NY, USA) (Lin et al., 2011). That being said, dynamic balance was assessed using the TUG test. In the TUG test, participants had to stand up on a chair (a 43.2-cm-high chair without arms), walk for $3 \mathrm{~m}$ as briskly as possible, crossing a line marked on the floor, turning around, and then walk back to the chair before sitting on it again (Rees et al., 2007). Mobility and lower extremity performance were evaluated by 5 -repetition chair-rising, 6-m tandem gait, and 10-m walking tests (Rahimi et al., 2011; Rees et al., 2007). Apart from that, leg isometric strength was measured by a standard leg press dynamometer (Torvinen et al., 2002). Participants were allowed to do three attempts and the median value was used as the final score. The measurements were done in baseline and after 8 weeks of intervention in all interventions and control groups.

\section{Statistical analysis}

Statistical analyses were performed by IBM SPSS Statistics ver. 22.0 (IBM Co., Armonk, NY, USA). Prior to data analysis, all variables were subjected to the normality test. Descriptive statistics of the variables were presented in means and standard deviations. The effects of intervention between the groups were tested using a one-way analysis of variance (ANOVA) that compared differences between the pretest and posttest, followed by the Tukey post hoc test. The within group differences were analyzed by paired $t$-test. That being said, the significance level was set at two-sided $P<0.05$. Subsequently, the data are expressed as the mean \pm standard deviation.

\section{RESULTS}

Data screening indicated that all related assumptions, including the normal distribution of dependent variables and sphericity (i.e., homogeneity of variance and covariance), were met. The anthro- 
pometric characteristics of the participants in each group are shown in Table 1.

Table 2 displays the results of intragroup differences as well as differences between groups in all variables at baseline and after 8 weeks.
The ANOVA from differences between posttest and pretest revealed that there were significant changes in static $(P=0.004)$ and dynamic ( $P=0.004)$ balance. According to the post hoc analysis, in comparison with the control group, the time of static balance for

Table 1. Demographic and baseline characteristics of participants in each group ( $n=42)$

\begin{tabular}{lcrrrr}
\hline Variable & WBV $(\mathrm{n}=11)$ & $\mathrm{MT}(\mathrm{n}=12)$ & WBV+MT $(\mathrm{n}=10)$ & Control $(\mathrm{n}=9)$ & $P$-value \\
\hline Age $(\mathrm{yr})$ & $66.58 \pm 5.80$ & $69.20 \pm 3.88$ & $67.80 \pm 5.88$ & $68.90 \pm 7.48$ & 0.720 \\
Height $(\mathrm{cm})$ & $170.90 \pm 4.90$ & $166.60 \pm 6.88$ & $164.90 \pm 4.97$ & $170.20 \pm 5.02$ & 0.120 \\
Body weight $(\mathrm{kg})$ & $80.45 \pm 10.97$ & $72.07 \pm 6.00$ & $72.88 \pm 8.11$ & $78.86 \pm 8.61$ & 0.076 \\
BMl $\left(\mathrm{kg} / \mathrm{m}^{2}\right)$ & $27.45 \pm 2.64$ & $26.17 \pm 3.81$ & $26.79 \pm 2.69$ & $27.25 \pm 2.89$ & 0.771 \\
\hline
\end{tabular}

Values are presented as the mean \pm standard deviation. All values are in physiological range and are not significantly different from each other.

WBV, whole body vibration; MT, mental training; BMI, body mass index.

Table 2. Balance, functional performance, and leg isometric strength at baseline and after 8 weeks in the four analyzed groups

\begin{tabular}{|c|c|c|c|c|c|}
\hline Parameter & WBV & MT & WBV+MT & Control & $P$-value \\
\hline Postural stability (sec) & & & & & 0.004 \\
\hline Baseline & $2.22 \pm 0.79$ & $2.56 \pm 1.28$ & $3.15 \pm 1.96$ & $1.87 \pm 0.57$ & \\
\hline 8Weeks & $1.70 \pm 0.66$ & $1.36 \pm 0.36$ & $1.64 \pm 0.53$ & $2.20 \pm 0.60$ & \\
\hline Difference & $-0.51 \pm 0.58^{*}$ & $-1.20 \pm 1.16^{*}$ & $-1.51 \pm 1.82^{*}$ & $0.33 \pm 0.57$ & \\
\hline$P$-value & 0.011 & 0.028 & 0.011 & 0.121 & \\
\hline Timed Up and Go (sec) & & & & & 0.004 \\
\hline Baseline & $6.10 \pm 0.92$ & $6.47 \pm 0.71$ & $6.75 \pm 1.32$ & $5.82 \pm 1.06$ & \\
\hline 8 Weeks & $5.28 \pm 0.84$ & $5.76 \pm 0.72$ & $5.87 \pm 1.14$ & $5.82 \pm 0.90$ & \\
\hline Difference & $-0.82 \pm 0.48^{* * *}$ & $-0.71 \pm 0.52^{* *}$ & $-0.88 \pm 0.68^{* *}$ & $0.05 \pm 0.71$ & \\
\hline$P$-value & 0.001 & 0.003 & 0.002 & 0.99 & \\
\hline 5-repetition chair-rising (sec) & & & & & 0.001 \\
\hline Baseline & $9.74 \pm 2.36$ & $9.68 \pm 2.26$ & $10.36 \pm 1.40$ & $9.67 \pm 2.35$ & \\
\hline 8 Weeks & $7.25 \pm 1.91$ & $7.45 \pm 2.69$ & $7.30 \pm 1.52$ & $10.28 \pm 2.43$ & \\
\hline Difference & $-2.49 \pm 1.92^{* *}$ & $-2.23 \pm 1.41^{* *}$ & $-3.05 \pm 1.60^{* * *}$ & $0.60 \pm 0.36$ & \\
\hline$P$-value & 0.001 & 0.001 & 0.001 & 0.068 & \\
\hline 6-m tandem gait (sec) & & & & & 0.001 \\
\hline Baseline & $6.51 \pm 1.58$ & $6.49 \pm 0.71$ & $6.69 \pm 0.76$ & $5.85 \pm 1.03$ & \\
\hline 8 Weeks & $5.65 \pm 0.77$ & $6.08 \pm 1.00$ & $5.63 \pm 0.51$ & $6.47 \pm 1.20$ & \\
\hline Difference & $-0.86 \pm 0.96^{*}$ & $-0.26 \pm 0.77$ & $-1.05 \pm 0.50^{* * *}$ & $0.62 \pm 0.72$ & \\
\hline$P$-value & 0.012 & 0.091 & 0.001 & 0.082 & \\
\hline 10-m walking (sec) & & & & & 0.003 \\
\hline Baseline & $22.57 \pm 3.94$ & $23.75 \pm 4.85$ & $21.97 \pm 5.65$ & $21.46 \pm 4.71$ & \\
\hline 8 Weeks & $15.26 \pm 3.51$ & $17.14 \pm 4.62$ & $14.50 \pm 6.06$ & $20.73 \pm 4.45$ & \\
\hline Difference & $-7.31 \pm 4.79 * * *$ & $-7.21 \pm 4.61^{* *}$ & $-7.46 \pm 5.15^{* *}$ & $-0.87 \pm 2.26$ & \\
\hline$P$-value & 0.001 & 0.001 & 0.001 & 0.298 & \\
\hline Leg isometric strength $(\mathrm{N} / \mathrm{m})$ & & & & & 0.001 \\
\hline Baseline & $95.83 \pm 13.29$ & $79.20 \pm 22.64$ & $77.96 \pm 26.68$ & $85.70 \pm 19.71$ & \\
\hline 8 Weeks & $110.58 \pm 12.79$ & $93.90 \pm 22.17$ & $96.50 \pm 34.27$ & $88.40 \pm 19.39$ & \\
\hline Difference & $14.75 \pm 12.52^{* *}$ & $14.70 \pm 5.30^{* * *}$ & $19.50 \pm 10.30^{* * *}$ & $2.70 \pm 1.64$ & \\
\hline$P$-value & 0.002 & 0.001 & 0.001 & 0.22 & \\
\hline
\end{tabular}

Values are presented as the mean \pm standard deviation.

WBV, whole body vibration; MT, mental training.

Analysis of variance was used to compare differences between the four groups. Intragroup differences was calculated using paired $t$-test and marked with asterisks: ${ }^{*} P<0.05$,

${ }^{* *} P<0.001,{ }^{* * *} P<0.000$. 
MP (2.56 to $1.36 \mathrm{sec}, P=0.022)$ and combination exercise ( 3.15 to $1.64 \mathrm{sec}, P=0.004)$ was significantly reduced, but not in the WBV group (2.22 to $1.7 \mathrm{sec}, P=0.32$ ). In case of dynamic balance, the post boc analysis showed that, in comparison with the control group, WBV (6.1 to $5.28 \mathrm{sec}, P=0.01$ ), MT (6.47 to 5.76 sec, $P=0.038$ ), and mixed (6.75 to $5.87 \mathrm{sec}, P=0.007$ ) interventions significantly improved in dynamic balance. There were no significant differences among the experimental groups. Moreover, the paired $t$-test analysis illustrated that, after 8 weeks of exercise, all exercise groups showed significant improvement in both static and dynamic balance (Table 2).

There were significant differences between groups in 5-repetition chair-rising $(P=0.001), 6-\mathrm{m}$ tandem gait $(P=0.001)$, and $10-\mathrm{m}$ walking $(P=0.003)$ tests. The post hoc test indicated that, in contrast with the control group, the performance of 5-repetition chair-rising test improved in WBV $(P=0.003)$, MP $(P=0.003)$, and concurrent $(P=0.003)$ groups. Likewise, at $6-\mathrm{m}$ tandem gait test, we observed a significant decrease in WBV $(P=0.001)$, MP $(P=0.019)$, and mix $(P=0.001)$ groups. Equally, the execution time of the 10-m walking test was decreased after 8 weeks of WBV $(P=0.008), \mathrm{MP}(P=0.013)$, and concurrent $(P=0.009)$ exercises. The analysis of intragroups by $t$-test indicated that the WBV exercise, MT, and combination of WBV and MT improved time of execution in 5-repetition chair-rising and 10-m walking tests. However, the MT exercise showed no change of time in the 6-m tandem gait test $(P=0.091)$, while the WBV $(P=0.01)$ and combination of WBV, and MT $(P=0.001)$ decreased in time of this test. Furthermore, there were no significant changes in the control group (Table 2). Leg isometric strength improved considerably after 8 weeks of exercise $(P=0.001)$. This difference was related to WBV $(P=0.015), \mathrm{MP}(P=0.021)$, and concurrent $(P=0.001)$ exercises in contrast with the control groups. Additionally, the paired $t$-test analysis confirmed that there was significant improvement in WBV (95.83 to $110.58 \mathrm{~kg}, P=0.001$ ), MP (79.20 to $93.9 \mathrm{~kg}, P=0.001$ ), and concurrent (77.96 to $96.5 \mathrm{~kg}$, $P=0.001)$ groups.

\section{DISCUSSION}

The primary finding of this study showed that body balance and walking performance were improved in response to WBV and MT exercises. The findings of this study, therefore, reject the aforementioned hypothesis as the WBV+MT did not pose a greater effect on selected parameters in comparison with single effects of WBV or MT.
A good number of studies have confirmed the effects of WBV as a mode of exercise on mobility and postural control (Bautmans et al., 2005; Bogaerts et al., 2007b; Bogaerts et al., 2011; Hiroshige et al., 2014; Kawanabe et al., 2007; Rees et al., 2007; Rees et al., 2009; Verschueren et al., 2004). WBV exercise stimulates the neuromuscular system and activates the leg and postural muscles through a phenomenon otherwise known as the so-called "tonic vibration reflex." In this way, WBV-induced muscle spindles activate 1a a motoneurons and eventually facilitate muscle contractions (Costantino et al., 2014; Rees et al., 2007; Rittweger, 2010; Von Stengel et al., 2012). Moreover, WBV-induced central nervous system stimulation is another reason for improvement in balance. Vibration oscillatory waves increase synchronization of motor units, cocontraction of synergist muscle, motivate inhibition of the antagonist muscles, coordinate contraction of agonist and antagonist muscles, and amplify firing rates of motor units (Bogaerts et al., 2007a; Costantino et al., 2014; Rittweger, 2010). These neural adaptations in the lower limbs play an important role in body balance and mobility.

In line with previous studies (Beauchet et al., 2010; Decety, 1996; Fansler et al., 1985; Grangeon et al., 2011; Hamel and Lajoie, 2005; Kalicinski and Lobinger, 2013), current findings confirm an MT-induced increase in balance and motor performance. However, there are inconsistencies in this context. Some studies have shown that mental practice improves balance and motor performance (Beauchet et al., 2010; Hamel and Lajoie, 2005; Kim et al., 2012), while other studies have concluded that mental practice alone has little or no effect on balance (Batson et al., 2007; Linden et al., 1989).

It was suggested that the MT engages structures of the brain that are involved in cognitive control and motion-planning. In other words, similar to physical practice, all stages of cognitive motor control include planning, programming, and readiness to execute movements are involved, but the difference is that the executive phase is inhibited in the mental practice workouts (Decety, 1996).

That being said, some theories, such as the psycho-neuromuscular, pseudo-motivational, and cognitive accounts (Choi et al., 2010; Driskell et al., 1994; Fansler et al., 1985; Hamel and Lajoie, 2005) justify the effectiveness of mental imagery in improving postural control and motor performance in elderly people. It seems that, among these theories, "psycho-neuromuscular mechanism" has gained much support and attention. According to this theory that was presented by Jacobson and other scholars, mental imagery exercise generates potential actions and slight contrac- 
tions in the muscles and muscle activity-induced kinesthetic dispatches feedback to brain centers and effects on these areas that eventually create reinforcement and coordination in the neuromuscular system (Choi et al., 2010; Driskell et al., 1994; Fansler et al., 1985; Hamel and Lajoie, 2005). In this regard, the neurophysiologic findings confirmed that physical training and MT activate the analogous nervous mechanism, although the extent of the efferent neural activity following MT is less than actual movement (Choi et al., 2010). Collectively, we can state that mental practice can presumably improve coordination of the neuromuscular system and amplification of agonist, antagonist, and synergist muscles and then develop mobility strategies, thus controlling the postural control and performance.

The secondary finding of our current study confirmed an increase in leg isometric muscle strength in WBV (15.4\%), MT (18.6\%), and mixture of WBV and MT (23.8\%) among older men. In this regard, a systematic review and meta-analysis study (Lau et al., 2011) reported that WBV exercise has seen noteworthy effects on leg extension isometric strength, knee extension dynamic strength, and functional measures of leg muscle strength among the elderly (Lau et al., 2011).

WBV-induced improvements in muscle strength and performance are typically based on neuromuscular expansion (Merriman and Jackson, 2009; Rittweger, 2010). Since our intervention was 8 weeks long, it seems that the observed increase in strength is reached due to neurological adaptations. This is despite the fact that we did not measure neural adaptation in this study. However, there was a reported increase in leg isometric and explosive strength following one year of WBV exercise in tandem with muscle hypertrophy among older people. They suggested that both neural and hypertrophic adaptations contribute to the increase in muscle strength (Bogaerts et al., 2007a).

Previous studies also revealed the presence of a strong relation between balance and strength. Wolfson et al. (1995), for instance, considered the effects of lower limb strength, walking, and balance on the prevalence of falling among nursing home residents. They reported that the higher the increase in strength, the fewer the falls. This proves that a strong relation exists between lower limb strength, balance, and walking (Lam et al., 2012; Rees et al., 2008; Wolfson et al., 1995). Since the aging process is associated with decline in muscle strength, it is possible that the increase in muscle strength can affect balance and functional performance in older population.

There are many reasons for discrepancies among studies that investigate WBV and MT effects with different protocols and methods on physical fitness and motor performance in older adults. It can point to differences in amplitude, frequency, intensity, volume, position of exposure on WBV device, dynamic or static exercise on WBV device, and activities that may affect the outcomes. In the MT context, there is more diversity in protocol and methods. MT exercise protocols include position of the participants, duration and number of MT trials, sequencing of MT, directed versus undirected MT, as well as the instruction mode and type. Another source of diversity returns to participant health status, age and sex.

In conclusion, we demonstrated the positive effects from 8 weeks of WBV and MT exercises on balance, muscle performance, and muscle strength in older men. However, we could not find additional effects from a combination of WBV and MT exercises. Based on the results and research literature, it seems that older adults who could not, or are unwilling to take part in routine exercise such as traditional resistance exercise can take advantage of WBV and MT exercises, which are effective, safe, simple, and low-cost modes of exercise for older adults to improve their health through decreases in and/or inversions in the development of Sarcopenia, loss of the muscle tissue as a natural part of the aging process, and other related disorders.

\section{CONFLICT OF INTEREST}

No potential conflict of interest relevant to this article was reported.

\section{ACKNOWLEDGMENTS}

The authors thank elderly people who attended as subjects. This study confirmed by Iranian Research Center on Healthy Aging (IRCHA) at the Sabzevar University of Medical Sciences, Sabzevar, Iran. The ethics committee of IRCHA approved this study (ethics number: 122/9462).

\section{REFERENCES}

Adsuar JC, Del Pozo-Cruz B, Parraca JA, Olivares PR, Gusi N. Whole body vibration improves the single-leg stance static balance in women with fibromyalgia: a randomized controlled trial. J Sports Med Phys Fitness 2012;52:85-91.

Batson G, Feltman R, McBride C, Waring J. Effect of mental practice combined with physical practice on balance in the community-dwelling elderly. Act Adapt Aging 2007;31:1-18. 
Bautmans I, Van Hees E, Lemper JC, Mets T. The feasibility of whole body vibration in institutionalised elderly persons and its influence on muscle performance, balance and mobility: a randomised controlled trial [ISRCTN62535013]. BMC Geriatr 2005;5:17.

Beauchet O, Annweiler C, Assal F, Bridenbaugh S, Herrmann FR, Kressig RW, Allali G. Imagined Timed Up \& Go test: a new tool to assess higher-level gait and balance disorders in older adults? J Neurol Sci 2010;294:102-106

Bogaerts A, Delecluse C, Boonen S, Claessens AL, Milisen K, Verschueren $\mathrm{SM}$. Changes in balance, functional performance and fall risk following whole body vibration training and vitamin $\mathrm{D}$ supplementation in institutionalized elderly women. A 6 month randomized controlled trial. Gait Posture 2011;33:466-472.

Bogaerts A, Delecluse C, Claessens AL, Coudyzer W, Boonen S, Verschueren SM. Impact of whole-body vibration training versus fitness training on muscle strength and muscle mass in older men: a 1-year randomized controlled trial. J Gerontol A Biol Sci Med Sci 2007a;62: 630-635.

Bogaerts A, Verschueren S, Delecluse C, Claessens AL, Boonen S. Effects of whole body vibration training on postural control in older individuals: a 1 year randomized controlled trial. Gait Posture 2007b;26:309316.

Choi JH, Choi Y, Nam KS, Cho IS, Hwang YT, Kwon YH. Effect of mental training on the balance control ability of healthy subjects. J Phys Ther Sci 2010;22:51-55.

Costantino C, Gimigliano R, Olvirri S, Gimigliano F. Whole body vibration in sport: a critical review. J Sports Med Phys Fitness 2014;54:757764.

Decety J. Do imagined and executed actions share the same neural substrate? Brain Res Cogn Brain Res 1996;3:87-93.

Driskell JE, Copper C, Moran A. Does mental practice enhance performance? J Appl Psychol 1994;79:481-492

Fansler CL, Poff CL, Shepard KF. Effects of mental practice on balance in elderly women. Phys Ther 1985;65:1332-1338.

Feltz DL, Landers DM, Becker BJ. A revised meta-analysis of the mental practice literature on motor skill learning. In: Druckmann D, Swets JA, editors. Enhancing human performance: issues, theories, and techniques. Washington, DC; National Academy Press; 1988. p. 61-101.

Goudarzian M, Rahimi M, Karimi N, Samadi A, Ajudani R, Sahaf R, Ghavi $S$. Mobility, balance, and muscle strength adaptations to short-term whole body vibration training plus oral creatine supplementation in elderly women. Asian J Sports Med 2017:8:e36793.

Grangeon M, Guillot A, Collet C. Postural control during visual and kinesthetic motor imagery. Appl Psychophysiol Biofeedback 2011;36:4756.
Hamel MF, Lajoie Y. Mental imagery. Effects on static balance and attentional demands of the elderly. Aging Clin Exp Res 2005;17:223-228.

Hiroshige K, Mahbub MH, Harada N. Effects of whole-body vibration on postural balance and proprioception in healthy young and elderly subjects: a randomized cross-over study. J Sports Med Phys Fitness 2014;54:216-224.

Hosseini SS, Rostamkhany H, Naghiloo Z. The effects of balance, mental and concurrent training on balance in healthy older males. J Res Rehabil Sci 2011;6:221-228.

Jackson PL, Doyon J, Richards CL, Malouin F. The efficacy of combined physical and mental practice in the learning of a foot-sequence task after stroke: a case report. Neurorehabil Neural Repair 2004;18:106111.

Kalicinski M, Lobinger BH. Benefits of motor and exercise imagery for older adults. J Imag Res Sport Phys Act 2013;8:61-75.

Kargarfard M, Lam ET, Shariat A, Asle Mohammadi M, Afrasiabi S, Shaw I, Shaw BS. Effects of endurance and high intensity training on ICAM1 and VCAM-1 levels and arterial pressure in obese and normal weight adolescents. Phys Sportsmed 2016;44:208-216.

Kawanabe K, Kawashima A, Sashimoto I, Takeda T, Sato Y, Iwamoto J. Effect of whole-body vibration exercise and muscle strengthening, balance, and walking exercises on walking ability in the elderly. Keio J Med 2007;56:28-33.

Kim BH, Newton RA, Sachs ML, Glutting JJ, Glanz K. Effect of guided relaxation and imagery on falls self-efficacy: a randomized controlled trial. J Am Geriatr Soc 2012;60:1109-1114.

Lam FM, Lau RW, Chung RC, Pang MY. The effect of whole body vibration on balance, mobility and falls in older adults: a systematic review and meta-analysis. Maturitas 2012;72:206-213.

Lau RW, Liao LR, Yu F, Teo T, Chung RC, Pang MY. The effects of whole body vibration therapy on bone mineral density and leg muscle strength in older adults: a systematic review and meta-analysis. Clin Rehabil 2011;25:975-988

Lin WH, Lin JD, Wang YT, Lee AJ. Effects of eight-week whole-body vibration training on postural stability in elderly adults. Port J Sport Sci 2011;11(Suppl 2):1019-1022.

Linden CA, Uhley JE, Smith D, Bush MA. The effects of mental practice on walking balance in an elderly population. OTJR: Occup Particip Health 1989;9:155-169.

Malouin F, Richards CL. Mental practice for relearning locomotor skills. Phys Ther 2010;90:240-251.

Malouin F, Richards CL, Durand A. Normal aging and motor imagery vividness: implications for mental practice training in rehabilitation. Arch Phys Med Rehabil 2010a;91:1122-1127.

Malouin F, Richards CL, Jackson PL, Doyon J, Guillot A, Collet C. Motor 
imagery for optimizing the reacquisition of locomotor skills after cerebral damage. Neurophysiol Found Ment Motor Imag 2010b:161-176.

Merriman $\mathrm{H}$, Jackson $\mathrm{K}$. The effects of whole-body vibration training in aging adults: a systematic review. J Geriatr Phys Ther 2009;32:134-145.

Osugi T, Iwamoto J, Yamazaki M, Takakuwa M. Effect of a combination of whole body vibration exercise and squat training on body balance, muscle power, and walking ability in the elderly. Ther Clin Risk Manag 2014;10:131-138.

Rahimi M, Kordi M, Karimi N, Gaeini A, Samadi A, Ali moradi N. The effect of whole body vibration training and creatine supplementation on lower extremity performance and balance in older men. Irainan J Aging 2011;6:38-46.

Rees S, Murphy A, Watsford M. Effects of vibration exercise on muscle performance and mobility in an older population. J Aging Phys Act 2007;15:367-381.

Rees SS, Murphy AJ, Watsford ML. Effects of whole-body vibration exercise on lower-extremity muscle strength and power in an older population: a randomized clinical trial. Phys Ther 2008;88:462-470.

Rees SS, Murphy AJ, Watsford ML. Effects of whole body vibration on postural steadiness in an older population. J Sci Med Sport 2009;12: 440-444.

Rittweger J. Vibration as an exercise modality: how it may work, and what its potential might be. Eur J Appl Physiol 2010;108:877-904.

Rogan S, Hilfiker R, Herren K, Radlinger L, de Bruin ED. Effects of whole- body vibration on postural control in elderly: a systematic review and meta-analysis. BMC Geriatr 2011;11:72.

Salmanian A, Farokhi A. Meta-analysis of done studies in field of mental practice of motor skills in iran. Olympic 2008;16:99-108.

Torvinen S, Kannus P, Sievänen H, Järvinen TA, Pasanen M, Kontulainen S, Järvinen TL, Järvinen M, Oja P, Vuori I. Effect of four-month vertical whole body vibration on performance and balance. Med Sci Sports Exerc 2002;34:1523-1528.

Verschueren SM, Roelants M, Delecluse C, Swinnen S, Vanderschueren D, Boonen S. Effect of 6-month whole body vibration training on hip density, muscle strength, and postural control in postmenopausal women: a randomized controlled pilot study. J Bone Miner Res 2004; 19:352-359.

von Stengel S, Kemmler W, Engelke K, Kalender WA. Effect of wholebody vibration on neuromuscular performance and body composition for females 65 years and older: a randomized-controlled trial. Scand J Med Sci Sports 2012;22:119-127.

Wolfson L, Judge J, Whipple R, King M. Strength is a major factor in balance, gait, and the occurrence of falls. J Gerontol A Biol Sci Med Sci 1995;50 Spec No:64-67.

Zhang L, Weng C, Liu M, Wang Q, Liu L, He Y. Effect of whole-body vibration exercise on mobility, balance ability and general health status in frail elderly patients: a pilot randomized controlled trial. Clin Rehabil 2014;28:59-68. 